TITLE: CARBON ATOM, DIMER AND TRIMER CHEMISTRY ON DIAMOND SURFACES FROM MOLECULAR DYNAMICS SIMULATIONS

AUTHOR(S): Steven M. Valone, MST-7

SUBMITTED TO: MRS Symposium on Modeling and Simulation of Thin-Film Processing, 1995 Spring MRS Meeting, San Francisco, CA 


\section{DISCLAIMER}

Portions of this document may be illegible in electronic image products. Images are produced from the best available original document. 


\title{
CARBON ATOM, DIMER AND TRIMER CHEMISTRY \\ ON DIAMOND SURFACES FROM MOLECULAR \\ DYNAMICS SIMULATIONS
}

STEVEN M. VALONE

Materials Science and Technology Division, Los Alamos National Laboratory, Los Alamos, NM 87545, USA

\begin{abstract}
Spectroscopic investigations of various atmospheres appearing in diamond film synthesis suggest evidence for carbon atoms, dimers or trimers. Molecular dynamics simulations with the Brenner hydrocarbon potential are being used to investigate the elementary reactions of these species on a hydrogen-terminated diamond (111) surface. In principle these types of simulations can be extended to simulations of growth morphologies, in the 1-2 monolayer regime presently.
\end{abstract}

\section{INTRODUCTION}

For more than a decade now molecular dynamics (MD) have been used to simulate reactions of gaseous species at solid surfaces. While these studies are not always quantitative, they can often lead to insights as to the behavior of these systems. The most compelling example to date perhaps is the etching of silicon by fluorine 1 .

In this spirit we examine the reactivity of carbon atoms, dimers and trimers on diamond (111) at $1200 \mathrm{~K}$. Each of these species has been observed spectroscopically in various atmospheres designed for diamond growth. The monatomic and triatomic species are mostly associated with flames and arc jets, ${ }^{2-4}$ whereas $C_{2}$ is thought to be prevalent in the microwave decomposition of buckministerfullerenes. ${ }^{5}$

The types of insights that we gain are: (1) Suggestions for new reactions, (2) Establish focus for more sophisticated investigations and (3) Estimates of rates when no others are available.

The ultimate goal for these types of investigates would be improved processes and improved process models where large-length or long-time scale phenomena are based on small-length or short-time scale submodels.

While the MD calculation itself is a deterministic one, the initial conditions for both the surface and the incoming gas molecule are sampled randomly. True to random sampling methods, we very quickly get a rough idea of the likelihood of major events. However, thoroughly quantifying any of these systems could cost a grad student his academic life.

\section{COMPUTATIONAL BACKGROUND}

MD simulations require that a choice be made as to how atoms interact in any given configuration. For hydrocarbons, an effective choice is the Brenner potential and specifically, for the specialists, potential III with torsional terms active. ${ }^{6}$ The Brenner 
potential is relatively unique in its ability to recognize different hybridization environments which is essential for these carbonaceous of systems.

Next a system of 160 carbon atoms in a diamond lattice is terminated with 16 hydrogen atoms. The (111) surface is exposed to the various gas species after several annealing steps.

To start a trajectory I use the following sequence of steps:

1. Anneal the pristine (111) surface

2. Choose a random position for the center of mass of the incoming gas atom or molecule

3. Choose a random set of velocities for the incoming species.

4. If the impinging species is a molecule, run 10 annealing steps to randomize the rotational and vibrational degrees of freedom

5. Run the full system trajectory

Typically for a system of $4 \times 4$ lattice constants the duration of the trajectory is far shorter than the interval between collisions. It is neither computationally feasible nor physically insightful to follow this intervening time. Step 1 has a second purpose, that being to substitute for the time interval between collisions. During that step, the system becomes randomized into a statistically independent state. This is akin to a numerical form of annealing.

\section{RESULTS}

We consider the results of each of the three species of interest, carbon monomers, dimers and trimers. They are only considered separately, never in combination, although they often appear that way in many practical systems. Our purpose here is to look at the reactivities of these three species exclusive of other complications.

$\underline{\mathrm{C} 1}$

As reported previously, 7 atomic carbon will not add to a pristine diamond (111) surface (based on 20 trajectories). See Figure 1.

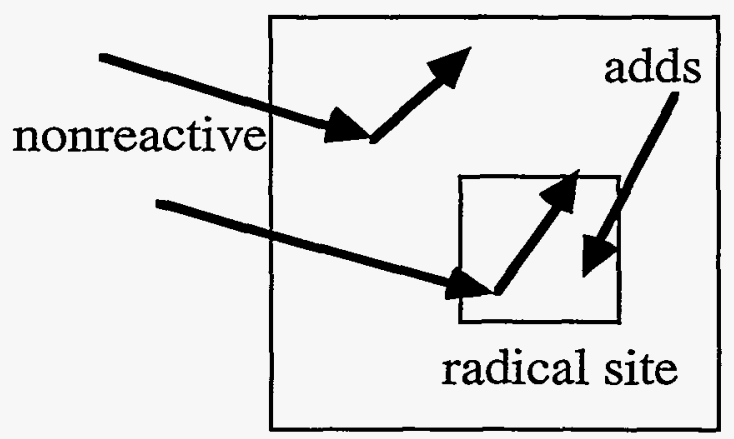

FIGURE 1. Schematic of a surface on which atomic carbon is impinging. The arrows are meant to represent trajectories of carbon atoms. Trajectories striking outside of the radical site (inner box) are always nonreactive. $85 \%$ of the trajectories striking the radical site are nonreactive, but the rest add to the surface. 
This seems likely because of the large difference in bond energy between a $\mathrm{CH}$ molecule ${ }^{8}$ and a $\mathrm{C}-\mathrm{H}$ bond in a diamond (111) surface. The later is around a volt more stable.

However if a radical site is somehow formed by another species, MD simulations with the Brenner potential estimate (based on 30 trajectories) that the carbon atom will add with probability 0.15 . If the radical sites are produced by atomic hydrogen, the probability for a reactive site being available is approximately 0.08 . Taking the product of these two probabilities gives the overall reaction probability of approximately 0.012 .

Once a carbon atom has added, presumably it quickly becomes saturated with hydrogen from incoming atomic and/or molecular hydrogen. How additional atomic carbon reacts with such surface species is unknown at this time. We restrict our attention here to elementary events.

As a sanity check, we estimate a growth rate for an atmospheric pressure thermal plasma. As estimated by $Y u$ and coworkers ${ }^{10}$ there is dependence of both carbon atom fraction, $x_{C}$, and growth rate on boundary layer thickness, $\delta$, as tracked in Table 1 . The contribution to growth rate due to atomic carbon, $\mathrm{r}_{\mathrm{G}, \mathrm{C}}$, is separated from the total, $\mathrm{r}_{\mathrm{G}, \text { total. }}$

Table 1. Predicted growth rates vs carbon atom near-surface concentrationa

\begin{tabular}{|r|r|r|r|r|}
\hline$\delta(\mathrm{mm})$ & \multicolumn{1}{|c|}{$\mathrm{X}_{\mathrm{C}}$} & \multicolumn{1}{c|}{$\mathrm{r}_{\mathrm{MD}} \mathrm{b}^{\mathrm{b}}$} & \multicolumn{1}{c|}{$\mathrm{r}_{\mathrm{G}, \mathrm{C}}$} & \multicolumn{1}{c|}{$\mathrm{r}_{\mathrm{G}, \text { total }}$} \\
\hline \hline 2.0 & $2.0(-7)$ & 0.11 & 0.15 & 10 \\
\hline 1.0 & $1.5(-5)$ & 8.2 & 10 & 50 \\
\hline 0.5 & $1.0(-4)$ & 55 & 100 & 200 \\
\hline 0.1 & $8.0(-4)$ & 437 & 650 & 650 \\
\hline
\end{tabular}

a All but $r_{M D}$ column from Reference 10.

b Present work.

First note that the growth rate from Reference 10 are for the (100) surface.

Nevertheless, using the hydrodynamically predicted values for $x_{C}$ and the MD predicted values of site specific reactivity, the MD predicted rates, $\mathrm{r}_{\mathrm{MD}}$, qualitatively track the reactive hydrodynamic prediction, $\mathrm{r}_{\mathrm{G}, C}$. Second note that the growth rates for (100) and (111) are typically with a factor of two or three of each other. Finally, Harris estimates probable errors in the hydrodynamically predicted rates of about a factor of one hundred. 10,11 In light of these comments, the relative success of the MD prediction is quite encouraging.

$\underline{\mathrm{C} 2}$

Much of the motivation for studying the reactivity of $\mathrm{C}_{2}$ comes from the work of Gruen and coworkers. 5 The assertion from that work is that it may be possible to synthesize diamond films from $\mathrm{C}_{2}$ precursors without adding large amounts of hydrogen to the reactive gas stream. The results of these simulations fully support this notion.

Unlike the carbon atom, the dimer needs no encouragement whatsoever to react on the diamond surface. Around $78 \%$ of the trajectories (based on 14 trajectories) result in 
some reactive event. However these fall into not one but at least four channels, as illustrated in Figures $2-4$ below.

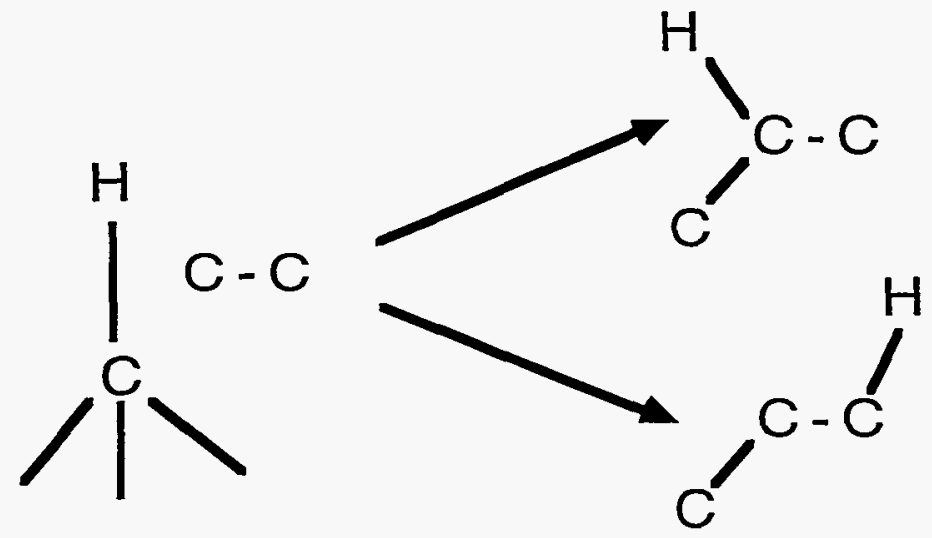

FIGURE 2. Insertion type reaction between $C_{2}$ and surface hydrogen. It is also possible for the hydrogen to migrate to the other carbon atom on the dimer.

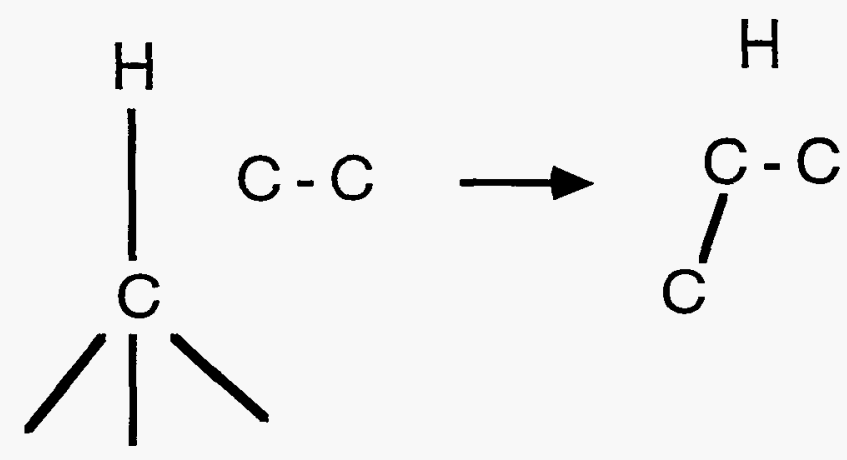

FIGURE 3. Substitution type reaction between $\mathrm{C}_{2}$ and surface hydrogen. The dimer replaces the surface hydrogen which is ejected back into the atmosphere.

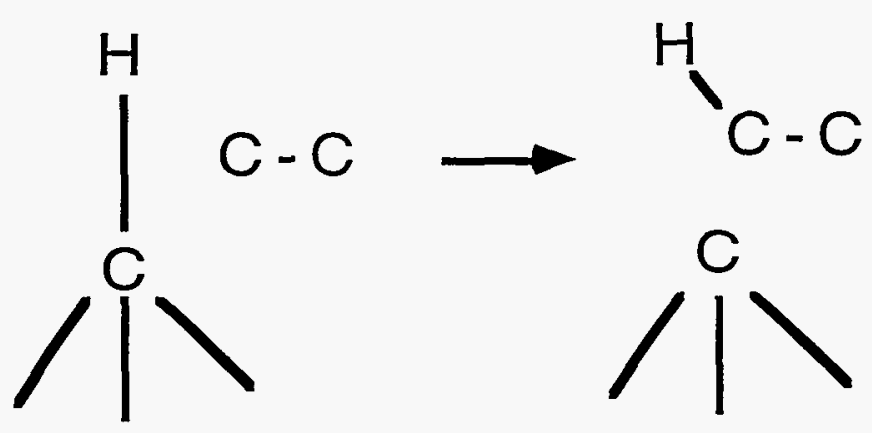

FIGURE 4. Abstraction type reaction between $\mathrm{C}_{2}$ and surface hydrogen. The dimer removes the surface hydrogen in much the same way that atomic hydrogen might. The resulting byproduct is an acyl or acetylenic radical.

The most prevalent channel is the insertion reaction which occurs with probability 0.33 . 
In this case, it is particularly important to note that $\mathrm{C}_{2}$ is part of the database used to parameterize the potential. For this reason there is some reason for optimism about the qualitative reliability of the results.

\section{$\underline{\mathrm{C} 3}$}

Here it is important to realize that the carbon trimer is linear. There is no ring opening energy release and the species must bend to achieve a tetrahedral configuration necessary for diamond and not graphitic growth.

So far only one reactive event (out of 4 trajectories) is observed for the trimer, a physisorbed looking arrangement. In fact the trimer seems to settle along a trough between two rows hydrogen atoms in the (100) direction. The adsorption occurs with probability 0.25 . The surface atoms appear essentially unperturbed which is the basis for calling this a physisorbed state.

The rest of the trajectories are nonreactive. Perhaps this is not surprising given that the trimer is thermodynamically more stable than the dimer.

\section{SUMMARY}

Spectroscopic investigations of various atmospheres appearing in diamond film synthesis suggest evidence for carbon atoms, dimers or trimers. Molecular dynamics simulations with the Brenner hydrocarbon potential are being used to investigate the elementary reactions of these species on a hydrogen-terminated diamond (111) surface.

As reported previously, the addition of carbon atoms to the surface requires the presence of a radical site such as that formed by hydrogen abstraction with atomic hydrogen. At about $1200 \mathrm{~K}$, the reaction probability (i.e. reactive sticking coefficient) on a (111) face is about 0.012 . Using hydrodynamically predicted values of near-surface carbon atom fractions for different operating conditions of an atmospheric pressure thermal plasma ${ }^{10}$ in conjunction with this value of the site-specific reactive, reasonable agreement with other predictions is obtained.

Carbon trimers stick with approximately probability 0.25 . The sticking arrange may be along a major crystallographic axis. No other surface atoms show significant displacement suggesting possibly some physisorbed state.

Carbon dimers on the other hand undergo a bewildering set of reactions. Nonreactive events only occur around $22 \%$ of the time. The most prevalent reactive evident is substitution of a surface hydrogen by the dimer. The displaced hydrogen can either be ejected or can become attached to the newly deposited dimer. In total, these simulations fully support the notion that carbon films, perhaps even diamond itself, may be deposited with $\mathrm{C}_{2}$ species, but without additional hydrogen.

For $\mathrm{C}_{2}$ it is very compelling to wonder if even in small amounts 3,4 it may be having a large influence on diamond growth. One can equally imagine controlling nucleation density by controlling the near-surface concentration of $\mathrm{C}_{2}$ during the synthesis. Perhaps the most important aspect of this work is that, with some of the key reaction channels identified by MD simulations, more quantitative methods such as high-level configuration interaction calculations or the most sophisticated density functional calculations, for evaluating the kinetics of this system. The type of model that one would want to use for this purpose is probably a transition state theory approach. 
Finally many other species reactivities can be explored with this approach including the classics, methyl radicals and acetylene molecules. Another curious minor species might be carbine radicals ${ }^{9}$. Moreover, any other surface of interest can be used in place of the (111) surface used here with the (100) being the most interesting.

\section{REFERENCES}

1 F. H. Stillinger and T. A. Weber, J. Chem. Phys. 88, 5123 (1988); Phys. Rev. Lett. 62, 2144 (1989);

P. C. Weakliem, C. J. Wu and E. A. Carter, Phys. Rev. Lett. 69, 200 (1992);

M.E. Barone and D. B. Graves, submitted.

2 G. A. Raiche, G. P. Smith and J. B. Jeffries, "Diagnostics of a Diamond-Depositing DC-Arcjet Plasma," in New Diamond Science and Technology 1991, ed. R. Messier, J. T. Glass, J. E. Butler, and R. Roy, (MRS, Pittsburgh, 1991), p. 251.

3 D. G. Goodwin, Appl. Phys. Lett. 59, 277 (1991).

4 Y. Matsui, A. Yuuki, M. Sahara and Y. Hirose, Japan. J. Appl. Phys. 28, 1718 (1989).

5 D. M. Gruen, S. Liu, A. R. Krauss and X. Pan, J. Appl. Phys. 75, 1758 (1994);

D. M. Gruen, S. Liu, A. R. Krauss, J. Luo and X. Pan, Appl. Phys. Lett. 64, 1502 (1994).

6 D. W. Brenner, Phys. Rev. B 42, 9458 (1990);

D. W. Brenner, J. A. Harrison, C. T. White and R. J. Colton,

Thin Solid Films 206, 220 (1991).

The different Roman numerals associated the potential represent different parameterizations of the same functional form. Potential III is generally regarded as the best compromise for the properties considered.

7 S. M. Valone, 1994 Gordon Research Conference on Diamond Growth.

8 K. P. Huber and G. Herzberg, Molecular Spectra and Molecular Structure IV. Constants of Diatomic Molecules, (van Nostrand Reinhold, New York, 1979), p140.

9 D. G. Goodwin and G. G. Gavillet, J Appl. Phys. 68, 6393 (1990).

10 B. W. Yu and S. L. Girshick, J Appl. Phys. 75, 3914 (1994).

11 S. J. Harris, Appl. Phys Lett.. 56, 2298 (1991);

S. J. Harris and D. G. Goodwin, J Phys Chem. 97, 23 (1993).

\section{DISCLAIMER}

This report was prepared as an account of work sponsored by an agency of the United States Government. Neither the United States Government nor any agency thereof, nor any of their employees, makes any warranty, express or implied, or assumes any legal liability or responsibility for the accuracy, completeness, or usefulness of any information, apparatus, product, or process disclosed, or represents that its use would not infringe privately owned rights. Reference herein to any specific commercial product, process, or service by trade name, trademark, manufacturer, or otherwise does not necessarily constitute or imply its endorsement, recommendation, or favoring by the United States Government or any agency thereof. The views and opinions of authors expressed herein do not necessarily state or reflect those of the United States Government or any agency thereof. 